\title{
对定量分析数学化的若干思考
}

甘峰*, 褚泳欣, 杨维岗

中山大学化学学院, 广州 510275

摘要: 对定量分析的数学化问题进行了初步的分析和讨论。通过对化学定量分析、仪器定量分析和化学计量学在建 立和应用数学理论方面的现状进行分析之后, 指出定量分析的数学化尚未完成。定量分析的数学化应该在基于化学 反应的基础上构建包含化学参数、自洽的、完备的数学方程, 进而发展为数学定理或定律才能实现。

关键词: 定量分析化学; 数学化

中图分类号: G64; O6

\section{Some Considerations on the Mathematization of Quantitative Analysis}

\section{Feng Gan *, Yongxin Chu, Weilan Yang}

School of Chemistry, Sun Yat-Sen University, Guangzhou 510275, China.

\begin{abstract}
This paper provides a primary analysis and discuss on the mathematization of quantitative analysis. By analyzing the present situation of chemical quantitative analysis, instrumental analysis and chemometrics in the establishment and application of mathematical theories, it pointed out that the mathematization of quantitative analysis has not been realized. The mathematization of quantitative analysis should be realized by establishing mathematical equation that contains chemical parameters, is self-contained and can be evolved into mathematical theorem.
\end{abstract}

Key Words: Chemical quantitative analysis; Mathematization

有人将分析化学尊为 “化学之母”, 以彰显其在化学学科的建立和发展过程起到的至关重要的作 用。然而也有人将其视为 “化学的婢女”, 以强调其在化学学科的建立过程中只起到辅助性的作用。 不同的人基于自己不同的 “利益” 取向, 由此对分析化学表现出不同的态度, 这本来是可以理解的。 但是, 由于不同的人所处的科学地位的不同, 他们的行为却在一定程度上延缓了分析化学的发展, 甚至在破坏分析化学的发展。例如, 国外的很多高校在 20 世纪取消了分析化学学科, 取而代之的是 将分析化学分拆到其他化学学科之中。中国也曾经出现过类似的思潮, 幸未最终发生。如何纠正曾 经发生在分析化学上的错误认识, 以及防止类似的事情再度发生, 是摆在每一位分析化学家面前的 一个重要问题。而重中之重, 则是要找到问题的根源之所在。

分析化学处于一个较为筳尤的地位有其历史渊源。其实不只是分析化学, 整个化学学科均处于 一个沜䢟地位, 究其历史可追溯到康德 ${ }^{[1]}$ 。康德认为 “...chemistry can't be anything more than a systematic art or experimental doctrine, never a science proper, because the principles of chemistry are merely empirical and can't be presented a priori in intuition...” 在康德看来, 化学由于过于经验化使其 不能成为 “理性的科学” 。在现代的科学家中, 认为化学不能成为独立学科的人也不少, 有些科学 
家则更为极端。例如狄拉克 ${ }^{[2]}$ 认为 “The underlying laws necessary for the mathematical theory of a large part of physics and the whole of chemistry are thus completely known, and the difficulty is only that exact applications of these laws lead to equations which are too complicated to be soluble.”

现代科学的一个显著特点是数学化, 这是迈向(康德所指的)理性的科学的必然途径。物理学家 一直致力于物理学的数学化并且取得了巨大的成功。物理学的某些成果, 如量子纠缠、引力波等概 念, 均是在物理理论数学化形式下从理论上首先得到并最终为实验所证实。化学如果视自己为科学 共同体中的一分子, 理应(也必将)遵循科学共同体的发展趋势, 实现化学的数学化。由于化学分为无 机化学、有机化学、物理化学和分析化学四个领域, 化学的数学化意味着这四个领域均实现数学化, 难度非常之大。2017 年, 俞汝勤院士 ${ }^{[3]}$ 在中国海南海口市举行的 International Congress on Analytical Sciences 会议上, 提出借助化学计量学实现分析化学数学化的思想, 这是对整个分析化学具有非常 重要影响的历史事件。

本文的目的是对分析化学的一个重要分支一一定量分析化学的数学化现状进行分析和讨论。定 量分析在整个分析化学中起到前承定性分析后启结构分析的作用, 其数学化进程对整个分析化学的 数学化起到至关重要的作用。由于定量分析事实上被划分为化学定量分析(主要是容量分析法)和仪 器定量分析, 我们将对二者进行分别的分析和讨论。我们期望能够回答若干问题, 例如定量分析化 学是否已经实现了数学化? 如果没有, 那么又该如何实现其数学化? 化学计量学能否帮助定量分析 实现数学化? 作者认为定量分析的数学化问题, 不仅仅是分析化学自身理论发展的需要, 也是实现 分析化学整体的数学化, 乃至化学整体的数学化的必经之路, 具有非常重要的科学意义。

\section{1 化学定量分析的数学化现状}

化学定量分析法主要是指经典分析化学中的容量分析法, 它依据溶液中的化学反应为基础进行 定量分析。在化学定量分析中, 化学反应组分之间的化学计量关系起到决定性的作用。待测物必须 直接或者间接地与某个标准试剂(通常称为滴定剂) 以固定的化学反应计量关系进行反应, 其含量才 能通过消耗的标准试剂的量进行计算。无论涉及的反应的数目和复杂程度如何, 由于化学反应中组 分之间固有的化学计量关系不变, 因而多数情况下可以建立待测组分含量与标准试剂用量之间准确 的数学方程, 从而计算出待测组分的含量。正因为如此, 化学定量分析法也被称为是绝对定量分析 方法 ${ }^{[4]}$ 。以 $\mathrm{NaOH}$ 滴定 $\mathrm{HCl}$ 这个最简单的定量分析为例, 化学反应式如下:

$$
\mathrm{NaOH}(\mathrm{aq})+\mathrm{HCl}(\mathrm{aq})=\mathrm{NaCl}(\mathrm{aq})+\mathrm{H}_{2} \mathrm{O}(\mathrm{aq})
$$

由于反应的计量关系为 $1: 1$, 所以达到计量点时可以用下式表述:

$$
c(\mathrm{HCl}) \times V_{0}=c(\mathrm{NaOH}) \times V_{\mathrm{sp}}
$$

这里, $c(\mathrm{HCl})$ 和 $c(\mathrm{NaOH})$ 分别为 $\mathrm{HCl}$ 和 $\mathrm{NaOH}$ 的初始浓度; $V_{0}$ 是 $\mathrm{HCl}$ 的初始体积, $V_{\mathrm{sp}}$ 是计量点时 加入的 $\mathrm{NaOH}$ 的体积。

式(1)即等同于物理学中的杜杆平衡, 而加入的 $\mathrm{NaOH}$ 的摩尔数即等同于砝码质量。然而, 式(1) 自身并没有提供直接的方法来指示何时达到计量点(即杜杆的平衡点)。通常情况下, 容量分析法在 反应体系内加入化学指示剂, 利用其变色来指示滴定终点, 以此对计量点进行估计。由于对指示剂 的变色的判定因人而异, 这让化学定量分析法看上去更像一门技艺而非科学。更为糟糕的是, 指示 剂本身实际上也会参与到定量反应过程中去, 并改变了化学计量点的位置 ${ }^{[5]}$ 。打个通俗的比喻, 指 示剂实际上扮演了 “运动员” 和 “裁判员” 的双重身份, 因而它很难做到客观地指示化学计量点。

化学定量分析中的通常做法是以指示剂的影响小从而使得最终产生的误差可忽略为理由将指示 剂的影响不计入到方程(1)的理论框架中。但这种人为地将本来就包含的因素强行剥离出去的做法也 使得化学定量分析的理论框架不完善。当前, 虽然可以建立严格的数学方程去描述滴定过程 ${ }^{[6,7]}$, 但 是, 这些方程本身并没有很好地解决计量点的指示问题。并且, 这些滴定方程更多地用于描述滴定 过程, 而不是直接用于定量分析。 
国外一些分析化学教科书 ${ }^{[8]}$ 介绍了采用对滴定曲线求导的方式对化学计量点进行估计, 但未给 出严格的数学证明。在实际的定量分析中, 这种做法也很少见有实施。更多见到的是基于仪器测量 采用 Gran 图法 ${ }^{[9]}$ 对化学计量点进行估计, 一些自动滴定仪也采用了这种方法。采用 Gran 图的方法 因不会显著地介入到反应过程中, 被认为是优于采用指示剂的方法。然而, Gran 图法的数学模型并 不准确。仍以 $\mathrm{NaOH}$ 滴定 $\mathrm{HCl}$ 的反应为例, $\mathrm{Gran}$ 是从下式开始进行数学推导过程(即参考文献[9]中 的方程(1)):

$$
\left[\mathrm{H}^{+}\right]=c(\mathrm{HCl}) \times \frac{V_{0}}{V_{0}+V_{\mathrm{t}}}-V(\mathrm{NaOH}) \times \frac{V_{\mathrm{t}}}{V_{0}+V_{\mathrm{t}}}
$$

这里, $\left[\mathrm{H}^{+}\right]$是加入 $V_{\mathrm{t}}$ 体积的 $\mathrm{NaOH}$ 后体系的氢离子浓度。

如果在化学计量点的位置加入的 $\mathrm{NaOH}$ 体积为 $V_{\mathrm{sp}}\left(V_{\mathrm{t}}=V_{\mathrm{sp}}\right)$, 由于有 $c(\mathrm{HCl}) \times V_{0}=c(\mathrm{NaOH}) \times$ $V_{\mathrm{sp}}$, 所以从式(2)得到 $\left[\mathrm{H}^{+}\right]=0$ 。然而, 化学事实表明上述的反应在化学计量点时体系的氢离子的浓 度实际上为 $\left[\mathrm{H}^{+}\right]=1.00 \times 10^{-7} \mathrm{~mol} \cdot \mathrm{L}^{-1}$ 。这表明, Gran 图法的理论误差会达到 $\left(0-1.00 \times 10^{-7}\right) / 1.00 \times$ $10^{-7}=-100 \%$ 。显然, Gran 图的数学模型并不能令人满意。

所以, 不能认为已经建立了化学定量分析完备的、自洽的数学理论体系。由于化学定量分析有 逐步被仪器定量分析取代的趋势, 目前很少人将其作为重要的研究领域去深入探索, 相关的数学理 论方面的研究未见有重大的进展。有趣的是, 依附于滴定分析构建起来的滴定曲线方法, 却越来越 多地在物理和化学参数的测量方面发挥着作用。限于篇幅, 这里不做进一步讨论。

\section{2 仪器定量分析的数学化现状}

仪器分析法的引入对分析化学产生了巨大的促进作用, 也带来了负面的影响。由于仪器分析法 所采用的测量原理通常源自物理学, 因而也被称为是物理分析法。更为极端的情况是, 正因为仪器 分析法的这个特性, 导致 20 世纪 60 年代左右在美国的一些著名的大学中取消了分析化学的职位, 理由居然是 “它只是应用物理化学而已”。

如果把视线转到定量分析领域, 由于仪器定量分析法采用了物理学原理并辅之以一定的数学形 式, 似乎从一开始就实现了数学化。以原子吸收法为例, Walsh ${ }^{[10]} 1955$ 年提出了利用原子吸收效 应来进行定量分析的理论基础, 其数学方程如下:

$$
K_{\max }=\frac{2 \lambda^{2}}{D_{\lambda}} \sqrt{\frac{\ln 2}{\pi}} \times \frac{\pi e^{2}}{m c^{2}} N f
$$

这里, $K_{\max }$ 是吸收系数的峰值; $\lambda$ 是波长; $D$ 是多普勒变宽; $e$ 是电子的电荷; $m$ 是电子的质量; $c$ 是光速; $N$ 是原子的数目; $f$ 是振子强度。

式(3)表明原子吸收系数峰值与原子数目成正比, 也就是与组分浓度成正比。由于该方程中其他 相关的量在一定条件下是常数, 而 $f$ 可以通过标准样品得到, Walsh 由此推测原子吸收法有可能成 为绝对定量分析方法(文献中用词 absolute analysis), 即只需要通过测量值 $K_{\max }$ 直接计算出 $N$ 。

然而, 实际的情况是式(3)并没有被直接用于绝对定量分析。具体原因非常多, 此不赘述。迄今 为止, 仪器定量分析法基本上均采用一种称为校正的技术, 即用待测物的标准品配制一系列标准溶 液并测量其信号强度, 由此建立浓度与测量信号强度的线性方程, 其一般形式如下:

$$
y=k c+\eta+\varepsilon
$$

这里, $y$ 是测量信号强度; $k$ 是回归系数; $c$ 是组分浓度; $\eta$ 是与仪器状态及分析条件相关的量; $\varepsilon$ 是 与随机误差相关的量。未知样品在同一仪器和同样分析条件下测量其信号强度之后, 可以通过式(4) 计算出其浓度。

然而, 如果对仪器定量分析进行仔细的思索就会发现, 它确实不再基于化学反应理论。虽然化 学反应在样品处理和生成产物等阶段依然发挥着作用, 但是化学反应的计量平衡关系不再用于定量 分析。所以, 将仪器定量分析划分为物理分析并不为过, 毕竟关键的理论还是物理学原理。这也就 
不难理解 Liebhafsky 会感慨地说 “Like it or not, the chemistry is going out of analytical chemistry” [11]。

仪器定量分析的关键在于应用式(4), 它本身是一个纯粹的数学方程, 不包含任何的物理或化学 体系的参数, 将其视为物理分析和化学分析的基础理论显然均不能成立。并且, 测量得到的信号强 度是相对量而不是绝对量, 不能从测量信号强度本身直接计算出物质的摩尔数, 所以用式(4)作为仪 器定量分析的数学基础本身也不完备。综上所述, 我们尚不能认为仪器定量分析的数学化已经完成。

从仪器定量分析的发展动态来看, 从早期的利用式(4)建立一元线性回归方程, 已经过渡到了基 于化学计量学的多元校正理论实现多元线性回归、主成分回归、偏最小二乘回归等。而所涉及的仪

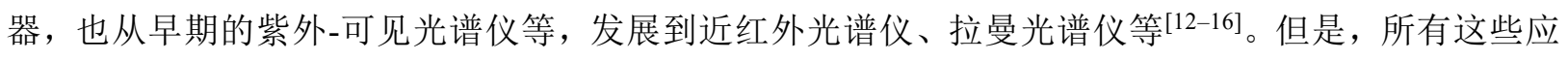
用都可视为是式(4)的扩展, 不能从本质上改变仪器定量分析没有实现数学化的现状。

\section{3 化学计量学对定量分析数学化的作用}

化学计量学 ${ }^{[17-19]}$ 被引入到分析化学之后对分析化学的诸多领域产生了深远的影响, 尤其是在定 量分析领域。例如, 近红外光谱技术和拉曼技术与化学计量学相结合之后, 构建出新的定量分析方 法 ${ }^{[20,21]}$, 极大地改变了传统的定量分析方式。而张量类型的数据的构建和多维分辨算法的引进和建

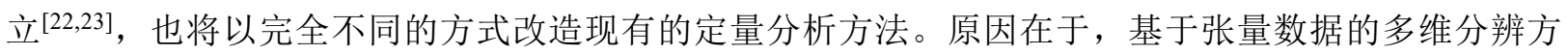
法从理论上可以得到唯一解 ${ }^{[23]}$, 这意味着基于张量数据在理论上是可以得到化学体系真实的光谱信 息和浓度信息，这将使得定量分析基于体系真实信息的基础上而不是抽象因子的基础上。

然而, 如果细致分析会发现, 采用这条途径依然无法达到理想的目标。例如, 如果基于二维荧 光技术得到的张量信号 $\underline{\mathbf{X}}$ 可以用三线性模型描述 ${ }^{[22]}$, 即:

$$
x_{i j k}=\sum_{n=1}^{N} a_{i n} b_{j n} c_{k n}+e_{i j k}, \quad i=1,2, \ldots, I ; j=1,2, \ldots, J ; k=1,2, \ldots, K
$$

这里, $x_{i j k}$ 是三维阵列 $\underline{\mathbf{X}}$ 的第 $i j k$ 个元素; $N$ 是有信号响应的组分数; $a_{i n}$ 是矩阵 $\mathbf{A}_{I N}$ 的第 in 个元素; $b_{j n}$ 是矩阵 $\mathbf{B}_{J N}$ 的第 $j n$ 个元素; $c_{k n}$ 是矩阵 $\mathbf{C}_{K N}$ 的第 $k n$ 个元素; $e_{i j k}$ 是残差三维阵列 $\underline{\mathbf{E}}$ 的第 $i j k$ 个元素。 这里的矩阵 $\mathbf{C}_{K N}$ 又称为浓度矩阵, 其每一列是一个组分的浓度向量。

在不存在光谱相似性和浓度共线性的情况下, 从模型(5)中可以得到唯一解, 从化学的角度可以 认为是得到体系中组分的真实光谱以及真实浓度。吴海龙等 ${ }^{[24]}$ 的研究工作也表明, 确实可以从该模 型中分解得到目标组分的苂光光谱, 且与对应组分的实际苂光光谱形状相同。这是否意味着同时得 到的浓度向量就是体系中组分的真实浓度变化呢? 真实的情况并非如此。分解得到的浓度向量中的 元素并非是真实的浓度值, 而只是与真实浓度成比例的值, 还必须采用校正的方法才能真正得到体 系组分的真实浓度值。这样一来, 基于化学计量学的张量理论得到的 “真实” 信息, 最终也不能实 现绝对定量分析, 而必须重新回到校正的老路上去。所以, 化学计量学尚未推动定量分析实现数学 化。

\section{4 结语}

总体而言, 定量分析的数学化当前还处在概念阶段, 当前尚无定论。从上面的讨论中可以看到, 定量分析在应用数学理论方面有了巨大的发展, 这使得它初步形成了理性的科学的形态。如何实现 定量分析的数学化仍是需要探讨的问题。但是应该认识到在定量分析中简单地引入(或者改造)现有 的数学理论不能视为是定量分析化学数学化的正确道路。从上面的分析中也可以看到这条路未必能 够走通。化学定量分析的数学化似乎应该在基于化学反应理论的基础上去构建具有化学反应内禀变 量的数学理论, 这个理论还必须是完备的、自洽的, 并且能够在某些条件下升华为定理或归结为定 律。如果定量分析数学定理能够构建成功, 则对于定量分析的数学化显然具有最大的支持效果。从 这一点上看, 容量分析法在将化学反应和数学相结合构建数学理论体系方面实际上是有成效的, 放 弃容量分析理论而仅依靠仪器定量分析去寻找定量分析的数学化未必是正确的道路。这也提示我们, 当前的分析化学教学中, 对于容量分析理论的教学不但不应该减少, 反而更应该加强。而对于其内 
容体系也应该做更为深入的拓展。例如, 当前的大多数分析化学教材中的容量分析部分, 宜将有关 酸碱滴定通式和配位滴定通式的内容加入。与此同时, 对于滴定体系的内容, 宜从以往的只关注待 测物的浓度变化, 过渡到对滴定体系中所有组分的浓度进行准确的数学描述。我们预期, 如果定量 分析的数学得以实现, 则定量分析有可能进入一个新的境界。

\section{参 考 文 献}

[1] Kant, I. The Metaphysical Foundation of Natural Science; Translator Bax, E. B.; Createspace Independent Publishing Platform: USA, 2015.

[2] Dirac, P. A. M. Proc. R. Soc. London, Ser. A 1929, 123, 714.

[3] Yu, R. Q. Proceeding of International Conference on Analytical Science; Chinese Chemical Society and International Union of Pure and Applied Chemistry: Beijing, 2017.

[4] Karayannis, M. I. Anal. Bioanal. Chem. 2011, 400, 3181.

[5] 李莲云, 余惠娟, 尹华勤, 甘峰. 大学化学, 2007, 22 (6), 54.

[6] De Levie, R. Anal. Chem. 2011, 400, 3181.

[7] 甘峰. 大学化学, 2007, 22 (5), 54.

[8] Harvey, D. Modern Analytical Chemistry; McGraw-Hill Higher Education: Boston, USA, 2000.

[9] Gran, G. Acta Chem. Scand. 1950, 4, 559.

[10] Walsh, A. Spectr. Acta. 1955, 7, 108.

[11] Laitinen, H. A. Anal. Chem. 1980, 52 (6), 605A.

[12] Xu, L.; Goodarzi, M.; Shi, W.; Cai, C. B.; Jiang, J. H. Chemom. Lab. Intel. Syst. 2014, 139, 58.

[13] Lin, Y. W.; Deng, B. C.; Xu, Q. S.; Yun, Y. H.; Liang, Y. Z. Chemom. Lab. Intel. Syst. 2016, $150,58$.

[14] Kong, J. J.; Chen, Z. P.; Chen, Y.; Yan, X. F.; Yu, R. Q. Chemom. Lab. Intel. Syst. 2016, 151, 9.

[15] Shi, C. X.; Chen, Z. P.; Chen, Y.; Liu, Q.; Yu, R. Q. Chemom. Lab. Intel. Syst. 2017, 169, 87.

[16] Kuang, Q. F; Li, Y. Z.; Wu, Y. M.; Li, R.; Dong, Y. C.; Xiong, Q.; Huang, Z. Y.; Li, M. L. Chemom. Lab. Intel. Syst. 2017, 162, 104.

[17] Wold, S. Chemom. Lab. Intel. Syst. 1995, 30, 109.

[18] Wold, S.; Sjostrom, M. Chemom. Lab. Intel. Syst. 1998, 44, 3.

[19] Kvalheim, O. M. J. Chemom. 2012, 26 (6), 210.

[20] Chen, Z. P.; Fevotte, G.; Caillet, A.; Littlejohn, D.; Morris, J. Anal. Chem. 2008, 80 (17), 6658.

[21] Li, C. Q.; Fang, Z. Y.; Xu, Q. S. Chemom. Lab. Intel. Syst. 2020, 198, 103935.

[22] Wu, H. L.; Shibukawa, M.; Oguma, K. J. Chemom. 1998, 12, 1.

[23] Rajko, R.; Omidikia, N.; Abdollahi, H.; Kompany-Zareh, M. Chemom. Lab. Intel. Syst. 2017, $160,91$.

[24] Zhu, L.; Wu, H. L.; Xie, L. X.; Fang, H.; Xiang, S. X.; Hu, Y.; Liu, Z.; Wang, T.; Yu, R. Q. Anal. Method 2016,8 (24), 4941. 